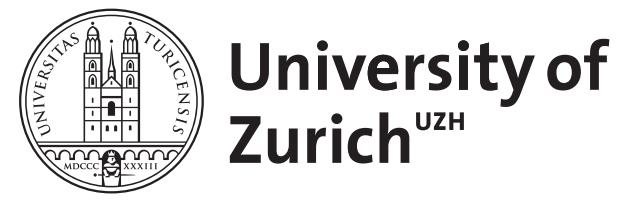

\title{
Are Inflammatory Cytokines Associated with Pain during Acute Myocardial Infarction?
}

Imholz, Laurin ; Meister-Langraf, Rebecca E ; Princip, Mary ; Fux, Michaela ; Schnyder, Ulrich ; Barth, Jürgen ; Znoj, Hansjörg ; Schmid, Jean-Paul ; von Känel, Roland

DOI: https://doi.org/10.1159/000481455

Posted at the Zurich Open Repository and Archive, University of Zurich ZORA URL: https://doi.org/10.5167/uzh-142631

Journal Article

Published Version

Originally published at:

Imholz, Laurin; Meister-Langraf, Rebecca E; Princip, Mary; Fux, Michaela; Schnyder, Ulrich; Barth, Jürgen; Znoj, Hansjörg; Schmid, Jean-Paul; von Känel, Roland (2017). Are Inflammatory Cytokines Associated with Pain during Acute Myocardial Infarction? Neuroimmunomodulation, 24(3):154-161. DOI: https://doi.org/10.1159/000481455 


\title{
Original Paper
}

Neuro[mmunoModulation

Neuroimmunomodulation 2017;24:154-161

DOI: $10.1159 / 000481455$

Received: June 21, 2017

Accepted after revision: September 12, 2017

Published online: November 4, 2017

\section{Are Inflammatory Cytokines Associated with Pain during Acute Myocardial Infarction?}

\author{
Laurin Imholz ${ }^{a}$ Rebecca E. Meister-Langrafa, e, Mary Princip $^{a, c, e}$ \\ Michaela Fux ${ }^{d}$ Ulrich Schnyder ${ }^{g}$ Jürgen Barth ${ }^{\text {h }}$ Hansjörg Znoj ${ }^{b}$ \\ Jean-Paul Schmidi Roland von Känel ${ }^{a, e, j}$ \\ ${ }^{a}$ Department of Neurology, Inselspital, Bern University Hospital, University of Bern, ${ }^{b}$ Division of Clinical Psychology \\ and Psychotherapy, Institute of Psychology, University of Bern, 'Preventive Cardiology and Sports Medicine, \\ and ${ }^{d}$ University Institute of Clinical Chemistry, Inselspital, Bern University Hospital, University of Bern, and \\ e Psychosomatic Research Group, Department of Clinical Research, University of Bern, Bern, ${ }^{f}$ Clienia Schlössli AG, \\ Oetwil am See, ${ }^{9}$ Department of Psychiatry and Psychotherapy and ${ }^{\mathrm{h}}$ Institute for Complementary and Integrative \\ Medicine, University Hospital Zurich, University of Zurich, Zurich, and Departments of 'Cardiology and \\ j.Psychosomatic Medicine, Clinic Barmelweid, Barmelweid, Switzerland
}

\section{Keywords}

Acute myocardial infarction - Cardiovascular disease . Pain - Cytokines - Inflammation - Posttraumatic stress . Psychological stress

\begin{abstract}
Objective: Pain and inflammation during acute myocardial infarction (AMI) have been associated with the development of posttraumatic stress disorder and may also impact negatively on somatic outcome. We investigated the relationship between pain during AMI and levels of circulating proinflammatory (tumor necrosis factor [TNF]-a, interleukin [IL]-6) and anti-inflammatory (IL-33 and tissue growth factor [TGF]- $\beta_{1}$ ) cytokines. Methods: Data were collected as part of the Myocardial Infarction - Stress Prevention Intervention (MISPRINT) study. We included 140 patients (mean age 59.6 years, $82.1 \%$ male) with high acute psychological distress within $48 \mathrm{~h}$ after Ml. Fasting blood samples were drawn thereafter to measure cytokine levels. Sociodemographic
\end{abstract}

\section{KARGER}

(C) 2017 S. Karger AG, Basel

E-Mail karger@karger.com

www.karger.com/nim factors, psychological and medical data, as well as cardiometabolic markers were assessed with questionnaires and patient interviews. Results: Linear regression models showed a significant positive correlation of pain with TGF- $\beta_{1}$ ( $b=770.91, p=0.031)$ and a significant inverse correlation of pain with IL-33 ( $b=-0.11, p=0.015)$ after controlling for age, gender, body mass index, lifetime depression, acute stress disorder symptoms, and the prognostic Global Registry of Acute Coronary Events (GRACE) score. Pain was not associated with IL- 6 but with the GRACE score $(b=0.01, p=0.003)$. Pain showed no significant association with TNF-a. Conclusion: Pain during MI was associated with anti- but not proinflammatory cytokines. As IL-33 has been shown to be cardioprotective, lower IL-33 levels with more intense pain may suggest a pathway through which increased pain during $\mathrm{MI}$ may have an impact on the medical prognosis.

(c) 2017 S. Karger AG, Basel

Clinical trial registration No. NCT01781247 (ClinicalTrials.gov).

Laurin Imholz, MMed

Department of Neurology, Inselspital, Bern University Hospital Freiburgstrasse 8

CH-3010 Bern (Switzerland)

E-Mail laurinimholz@gmail.com 


\section{Introduction}

Acute myocardial infarction (AMI) is a life-threatening event during which the majority of patients experience intense pain [1]. Pain intensity has been shown to be associated with peritraumatic psychological distress [1] and the development of posttraumatic stress in patients who suffered MI [2-5]. Further, pain intensity seems to be associated with the clinical outcome in the aftermath of MI. A previous study showed that patients who had experienced more pain during MI also had a higher risk of hospital readmission due to cardiovascular disease events during a 32-month follow-up [6]. Therefore, pain during MI may be associated with a poorer prognosis regarding mental and physical health.

Due to hypoxic tissue damage and cell death, several pro- and anti-inflammatory processes are triggered during AMI. In a first step, an inflammatory reaction serves to process and clear damaged tissue [7]. During this process, proinflammatory cytokines like tumor necrosis factor (TNF)- $\alpha$, interleukin (IL)-1, and IL-6 are expressed [8]. In a second step, inflammation suppression facilitates initiation of myofibroblast proliferation and scarring. Molecules of the transforming growth factor (TGF)- $\beta$ family play an important role in regulating this process $[8,9]$. Another cytokine that is involved in cardiac remodeling is IL-33: it reduces MI volume and improves contractile function [10]. Although proinflammatory effects have also been attributed to IL-33, IL-33 appears to be atheroprotective and might thereby oppose cardiovascular disease progression [11]. In this paper, we will discuss IL-33 as an anti-inflammatory cytokine based on investigations in cardiological patients.

Not only cardiac tissue injury, but also pain intensity and psychological distress may cause an increase in inflammation markers during AMI. Both acute and chronic psychosocial stress have been associated with the release of proinflammatory cytokines, e.g., TNF- $\alpha$ and IL-6 [12-15], and, as mentioned above, pain and acute psychological distress are correlated during AMI.

Proinflammatory cytokines have been associated with impaired psychological and physical health in the longterm, including posttraumatic stress [16-19]. In population-based studies, IL- 6 and TNF- $\alpha$ were both found to be associated with increased morbidity and mortality independent of other risk factors, particularly in men [20$22]$. Several studies showed an association between worse cardiovascular outcome and increased TNF- $\alpha$ [23] and IL-6 [24-26] levels specifically following an acute coronary syndrome (ACS). However, despite their anti-in-

Inflammatory Cytokines and Pain during AMI flammatory properties during MI, both IL-33 [27, 28] and TGF- $\beta$ [29] have been found to be associated with a worse prognosis in coronary artery disease.

To date, little is known about the interplay of pain and myocardial damage regulating inflammation in AMI. Since pain and inflammation are both predictors of future cardiovascular and psychological health, we examined their relationship during AMI. We hypothesized that stronger pain during AMI would be associated with increased levels of proinflammatory cytokines TNF- $\alpha$ and IL-6, independent of sociodemographic, medical, and psychometric data. Furthermore, we expected a negative association between pain and the anti-inflammatory TGF- $\beta_{1}$ and IL-33.

\section{Patients and Methods}

\section{Patients and Study Design}

The present study retrieved data from the randomized-controlled trial Myocardial Infarction - Stress Prevention Intervention (MI-SPRINT), which was conducted between 2012 and 2015. The trial included patients who were referred to the Inselspital, Bern University Hospital, with AMI, and aimed to test whether trauma-focused psychological counseling in this phase could reduce the development of posttraumatic stress [30]. The study protocol was approved by the ethics committee of the State of Bern, Switzerland, and written informed consent was obtained from all participants. In addition to confirmed ST elevation MI (STEMI) or non-STEMI, further inclusion criteria were age $\geq 18$ years, sufficient knowledge of the German language, stable circulatory conditions, and considerable pain and psychological distress during MI (cf. Psychometric Assessments). Specific exclusion criteria were severe comorbidity reducing life expectancy to $<1$ year, current severe depression per the cardiologist's clinical judgment, suicidal ideations during the last 2 weeks, cognitive impairment/disorientation, emergency coronary artery bypass graft surgery, and participation in another randomized, controlled clinical trial.

Within $48 \mathrm{~h}$ after hospital admission, participants underwent a standardized interview for the assessment of pain perception, medical history, and sociodemographic data. They also completed the Acute Stress Disorder Scale (ASDS). The following morning, a fasting blood sample was drawn to measure circulating biomarker concentrations.

Of 190 patients included, cytokine levels were assessable in 143. The most common reasons for missing values were the following: unexpected/immediate transfer to other hospitals, blood samples could not be processed on weekends, blood sampling was technically not possible, blood samples or values were lost, or blood samples were contaminated. In 3 cases, pertinent personal history data were lacking due to an unexpected transfer during their hospital stay. Data analysis could therefore be conducted on a final sample of 140 patients.

\section{Demographic and Medical Factors}

We obtained patient characteristics, including age, sex, living status, weight and height (to calculate the body mass index [BMI]); and medical history by standardized interview questions during

Neuroimmunomodulation 2017;24:154-161 
admission or from hospital charts. Medical information on the number of vessels involved in coronary lumen stenosis $>50 \%$, troponin $\mathrm{T}$ peak levels, left ventricular ejection fraction, and other parameters necessary to calculate the Global Registry of Acute Coronary Events (GRACE) score was retrieved from hospital charts.

\section{The GRACE Score}

The online tool "GRACE 2.0 ACS Risk Calculator" based on the revised GRACE algorithms for predicting death or death/myocardial infarction following an initial ACS [31] was used to calculate the GRACE score. The algorithm considers age, heart rate, systolic blood pressure, Killip class (indicating heart failure in AMI patients, [32]), initial serum creatinine concentration, positive initial cardiac markers, cardiac arrest on admission, and presence of ST deviation. A score is generated which is related to the occurrence of the named adverse outcomes with higher scores indicating higher risk of occurrence.

\section{Psychometric Assessment}

\section{Pain and Acute Psychological Distress}

Patients were asked to evaluate their most intense pain level experienced during AMI ("Please indicate how strong your pain was during the heart attack") on a scale from 0 to 10 , with 10 being the highest imaginable pain (visual analogue scale for pain [33]). For inclusion in MI-SPRINT, a score of at least 5 for experienced pain and of at least 5 for either fear of dying ("During my referral to the hospital, the emergency unit, or the intensive care unit, I was afraid I was dying") and/or helplessness ("When the doctor told me I had a heart attack, I was frightened, felt helpless, and was afraid of losing control of the situation") was required.

\section{ASD Symptoms}

The German version of the ASDS was used to assess symptoms of ASD $[34,35]$. The scale is a self-rating instrument with 19 items to be scored on a 5-point Likert scale, from 0 (not at all) to 4 (extremely). Sum scores range from 0 to 76 , with higher values indicating more stress. The ASDS consists of the 4 subscales dissociation, reexperiencing, avoidance, and arousal (DSM IV) [36]. All participants were asked to rate these symptoms with respect to MI. The instrument was validated in a cardiac sample and showed good internal consistency (Cronbach a sum score $=0.88$ ) [35]. Good reliability was also achieved in our sample (Cronbach $\alpha$ sum score $=0.84$ ).

\section{Laboratory Analysis}

Blood samples were collected into EDTA tubes and centrifuged for $10 \mathrm{~min}$ at 2,000 $\mathrm{g}$. Plasma was transferred to polypropylene tubes and stored at $-80^{\circ} \mathrm{C}$. Concentrations of biomarkers were determined using Luminex technology with magnetic bead-based immunoassays (human Th17 cytokine panel and TNF- $\alpha$, IL-6, IL33 , TGF- $\beta$, and TGF- $\beta_{1}$ assays; Bio-Rad Laboratories Inc., Hercules, CA, USA). Intra- and interassay coefficients of variation were $<10 \%$ for all biomarkers. Detection limits were $0.07 \mathrm{pg} / \mathrm{mL}$ (TNF- $\alpha$ ), $0.67 \mathrm{pg} / \mathrm{mL}$ (IL-6), $0.58 \mathrm{pg} / \mathrm{mL}$ (IL-33), and $3.9 \mathrm{pg} / \mathrm{mL}$ $\left(\mathrm{TGF}-\beta_{1}\right)$. The levels of lowest quantification (LLOQ) were as follows: $0.57 \mathrm{pg} / \mathrm{mL}$ (TNF- $\alpha$ ), $1.65 \mathrm{pg} / \mathrm{mL}$ (IL-6), $4.18 \mathrm{pg} / \mathrm{mL}$ (IL-33), and $1.69 \mathrm{pg} / \mathrm{mL}\left(\mathrm{TGF}-\beta_{1}\right)$. Fluorescence intensity results were used to distinguish nondetectable from nonquantifiable values. Values below sensitivity (not detectable) were substituted with half the detection limit. Values between sensitivity and LLOQ (not quantifiable) were substituted with half the LLOQ.
Table 1. Characteristics of the patients stratified by pain severity

\begin{tabular}{lrrrr}
\hline Variables & Total & \multicolumn{2}{c}{ Pain score } & \multirow{2}{*}{$p$} \\
\cline { 3 - 4 } & & $5-7$ & $8-10$ & \\
\hline Patients, $n$ & 140 & 52 & 88 & \\
$\quad$ \% & 100 & 37.1 & 62.9 & \\
Mean age, years & 59.6 & 58.7 & 60.1 & 0.47 \\
Female gender, \% & 17.9 & 11.5 & 21.6 & 0.13 \\
Mean BMI & 27.3 & 27.2 & 27.3 & 0.33 \\
Living alone, \% & 27.9 & 13.4 & 36.4 & $<0.01$ \\
Previous MI, \% & 8.6 & 5.8 & 10.2 & 0.36 \\
Depression, \% & 26.4 & 23.1 & 28.4 & 0.49 \\
Vessels involved, \% & & & & 0.28 \\
$\quad$ 0 vessels & 1.4 & 3.8 & 0 & \\
$\quad$ 1 vessel & 36.4 & 38.5 & 35.2 & \\
$\quad$ 2 vessels & 30.7 & 26.9 & 33.0 & \\
$\quad$ 3 vessels & 31.4 & 30.8 & 31.8 & \\
LVEF, \% & 47.4 & 47.6 & 47.3 & 0.89 \\
Troponin T, $\mu$ g/L & 4.0 & 3.3 & 4.3 & 0.11 \\
GRACE score & 107.6 & 106.3 & 108.4 & 0.82 \\
\hline
\end{tabular}

Peak troponin T and lifetime depression are listed. BMI, body mass index; GRACE, Global Registry of Acute Coronary Events; LVEF, left ventricular ejection fraction; MI, myocardial infarction.

\section{Statistical Analysis}

Data were analyzed using the PASW 21.0 statistical software package (SPSS Inc, Chicago, IL, USA). The level of significance was set at $p<0.05$ ( 2 tailed). Missing items were replaced using the expectation-maximization algorithm [37]. Concentrations of TNF- $\alpha$, IL-6, and IL-33 were log transformed to approximate a normal distribution. Multivariate normality of the data distribution was tested using Mahalanobis distance with a level of significance of $p<0.001$. To demonstrate patient characteristics, the sample was split into 2 groups with less (5-7 points) versus more intense pain (8-10 points). Pearson $\chi^{2}$ and independent-sample $t$ tests for categorical and continuous variables, respectively, were used to compare both groups on demographic and health characteristics. The Mann-Whitney $U$ test was used for group comparisons on BMI, GRACE score, and peak troponin level, as these values were not normally distributed.

We used multivariate linear regression with forced entry of covariates to test for an independent contribution of pain levels to the concentrations of the assessed cytokines. Variables were added to the model in 5 blocks. In a first step, we entered the a priori defined control variables (age and gender). Depression and BMI were entered in a 2 nd and the GRACE score in a 3rd step. In block 4, the ASDS sum score served as a psychometric control variable. Finally, pain was added to the model. For each cytokine, regression models were run separately. We did not adjust $p$ values for multiple comparisons because of the prespecified direction of the association between pain and cytokine levels.

Linearity, homoscedasticity, and absence of multicollinearity were tested by scatter plot and curve estimation. Durbin Watson statistics assured exclusion of autocorrelation. Results are expressed as unstandardized $b$ coefficients, standard errors of the mean, and changes in $R^{2}$ in each step with $p$ values. 


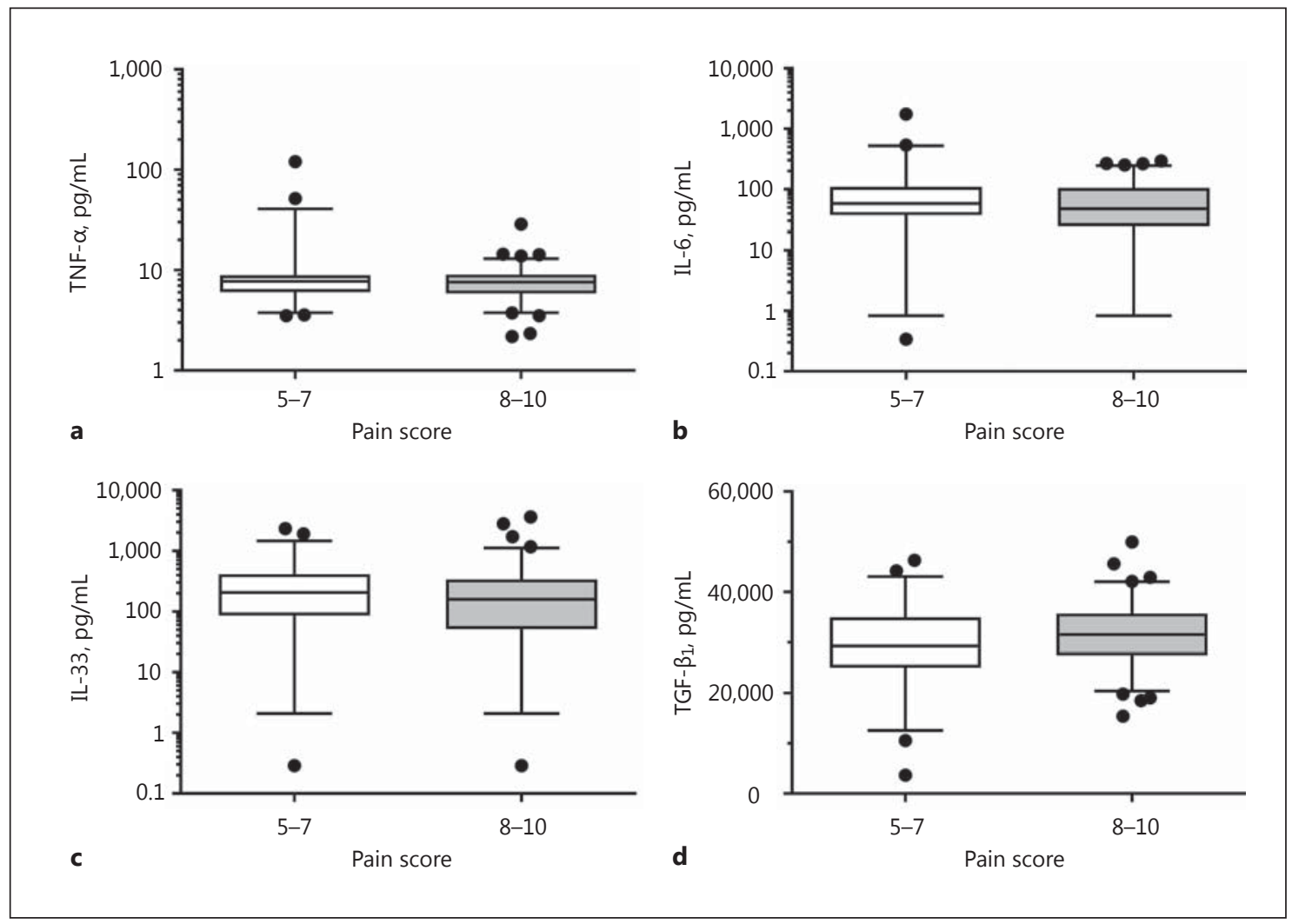

Fig. 1. Box plots of serum levels of cytokines TNF- $\alpha$ (a), IL-6 (b), IL-33 (c), and TGF- $\beta_{1}$ (d) grouped per less versus more intense pain (medians, interquartile ranges, whiskers at 5 th and 95 th percentiles).

Table 2. Cytokine assessments

\begin{tabular}{lrrrc}
\hline Results & TNF- $\alpha$ & IL-6 & IL-33 & TGF- $\beta$ \\
\hline Quantifiable, $n$ & 140 & 132 & 119 & 140 \\
Not quantifiable, $n$ & 0 & 7 & 19 & 0 \\
Not detectable, $n$ & 0 & 1 & 2 & 0 \\
\hline
\end{tabular}

\section{Results}

\section{Patient Characteristics}

Table 1 shows the patient characteristics of the total sample and both groups per pain level. The majority of patients were male. While few patients had had a previous MI, 1 out of 4 reported lifetime depression. The 2 groups did not differ significantly in age, gender distribution, and cardiac-related variables. Patients who experienced more pain were significantly more frequently living alone.
For illustrative purposes, cytokine values of both groups stratified according to the intensity of pain are shown in Figure 1. As shown in Table 2, most values were detectable.

\section{Regression Analysis}

Table 3 shows hierarchical regression models including the 4 cytokines as dependent variables.

$T N F-\alpha$. Only lifetime depression was significantly and inversely associated with TNF- $\alpha$ concentration $(p=$ $0.013)$. No significant association emerged for pain with TNF- $\alpha(p=0.45)$.

IL-6. Higher BMI $(p=0.006)$ and a higher GRACE score $(p=0.003)$ were both associated with higher IL-6 concentrations. Pain was not associated with IL-6 ( $p=0.23)$.

$I L-33$. Higher pain levels were significantly associated with lower IL-33 $(p=0.015)$. Pain explained an additional $4 \%$ of the variance in IL-33 concentration independently of all other covariates in the model. None of the other variables were significantly associated with IL-33.

Neuroimmunomodulation 2017;24:154-161 DOI: $10.1159 / 000481455$ 
Table 3. Regression analysis with TNF- $\alpha$, IL-6, IL-33, and TGF- $\beta_{1}$ as outcome variables

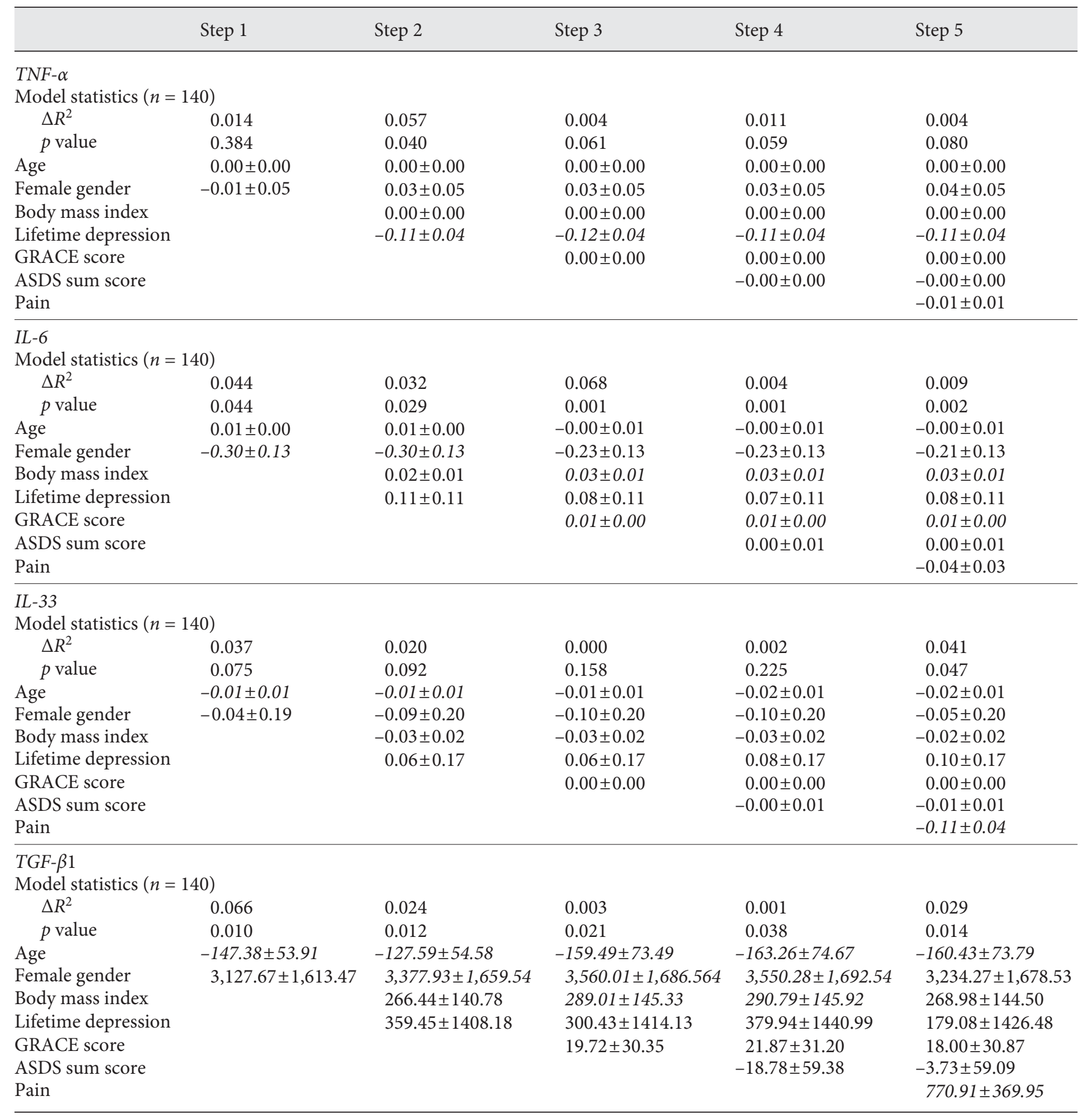

Data are shown as unstandardized $b$ coefficients (means \pm SEM). Results with $p<0.05$ are in italics. ASDS, Acute Stress Disorder Scale; GRACE, Global Registry of Acute Coronary Events. Values $<0.005$ were approximated to 0.00 . 
TGF- $\beta_{1}$. Higher pain levels were associated with a higher TGF- $\beta_{1}$ concentration $(p=0.031)$, whereas older patients had lower TGF- $\beta_{1}(p=0.039)$. Pain explained an additional $2.9 \%$ of the variance in TGF- $\beta_{1}$ concentration, independently of all other covariates in the model.

\section{Discussion}

The purpose of our study was to examine whether pain during AMI is related to inflammation, independent of demographic, medical, and psychometric factors. We found significant associations with levels of circulating anti-inflammatory cytokines but not with levels of proinflammatory cytokines.

More intense pain during MI was significantly associated with lower IL-33 levels. To the best of our knowledge, no study has previously examined circulating IL-33 during AMI and its relationship to pain or acute psychological distress. Since IL-33 was shown to have cardioprotective and anti-atherosclerotic properties in general [11], we interpret this relationship to reflect a path leading from more intense pain during AMI to poorer cardiac prognosis, although this assumption needs investigation in prospective studies. However, the potential consequences of lower circulating IL-33 levels during AMI are not unequivocal. While 2 studies found no prognostic significance of IL-33 [38, 39], 2 other studies in patients with STEMI reported increased mortality [28] and increased risk of recurrent MI [27] among those with high IL-33 levels. Further research is needed to evaluate the significance of decreased IL-33 in MI patients experiencing more pain.

We found a significant direct association between pain and the TGF- $\beta_{1}$ concentration. Only one study has previously examined TGF- $\beta$ during AMI and its prognostic significance [40]. That study showed neither a difference in circulating TGF- $\beta_{1}$ concentrations between ACS patients and patients with stable coronary artery disease nor an association with recurrent MI and mortality. An increase in TGF- $\beta_{1}$ during AMI with greater pain intensity can therefore be assumed to have no negative effect on prognosis. TGF- $\beta$ is known to be a significant mediator of nociception and to have protective effects against the development of chronic neuropathic pain [41]. The effect we found could therefore be part of a physiological counterregulation set off by acute pain.

We did not observe a significant association between pain and TNF- $\alpha$ during AMI. Since cardiac inflammation is also expected to produce TNF- $\alpha$ [8], a potential inde-

Inflammatory Cytokines and Pain during AMI pendent effect of pain or ASD on TNF- $\alpha$ levels might be superimposed by the acute phase response launched by myocardial damage.

We further found that neither pain nor ASD symptoms were significantly associated with IL-6 levels. A correlation, however, was observed for the GRACE score. This is in line with the previous literature showing that IL-6 is associated with increased morbidity and mortality after ACS [24-26]. We had hypothesized that pain or ASD would also be associated with IL-6, since it has previously been described as a predictor of posttraumatic stress disorder [42]. However, such a relationship may be too subtle compared to superimposed cardiac inflammation.

The characteristic course of the acute phase reaction during MI might be responsible for our nonsignificant results with regard to the proinflammatory cytokines. As mentioned above, cardiac inflammation during AMI can be divided in a first step, which is affected by proinflammatory cytokines and an inflammatory reaction to process and clear damaged tissue, and a second step, in which inflammation is suppressed by anti-inflammatory cytokines in order to initiate myofibroblast proliferation and scarring $[7,8]$. It is possible that, due to the delay between onset of symptoms and blood sampling, we primarily examined the second phase. Therefore, in our samples, proinflammatory cytokines may already have been downregulated by anti-inflammatory mediators and thereby escaped detection in relation to pain intensity.

Cytokines also showed associations with other demographic and medical variables. IL-6 was directly associated with BMI. This has been found previously in, for instance, the CoLaus study [43]. TGF- $\beta$ concentrations were lower in our younger study participants. Surprisingly, patients with lifetime depression had lower concentrations of TNF- $\alpha$ during AMI, although major depressive disorder has been associated with inflammation, including higher levels of TNF- $\alpha[44,45]$.

Our data indicated that patients experiencing more pain were significantly more often living alone. Other demographic variables, i.e., age, gender, and BMI, were not associated with pain. Cardiac markers like peak troponin value, the number of coronary vessels affected, left ventricular ejection fraction, and the GRACE score did also not differ between both groups (high vs. low pain indications). Pain was therefore not primarily influenced by cardiac injury, an interpretation which is supported by previous data showing that chest pain considerably relates to psychological factors [46].

Neuroimmunomodulation 2017;24:154-161 159 
Our study has several limitations. We measured cytokine levels only once, which does not trace modeling of their trajectory during the acute phase of MI. Blood samples could be drawn within several days after MI onset, depending upon how quickly patients were admitted to the hospital and included in our study. Consequently, as cytokine concentrations change over time during AMI, our results were affected by the variability of the interval between onset of pain and blood sampling. Recall bias is possible, as, due to the study design, we assessed pain retrospectively and only approached patients who were hemodynamically stable, often after the acute coronary intervention. We included patients who experienced a significant amount of pain, which reduces the variability in pain scores and thus the chance of finding a significant association with cytokine levels, while also limiting generalizability of our results to patients with little or no pain. Due to the cross-sectional design of our study, we are unable to draw causal inferences. Cytokines have previously been discussed as transmitters of danger signals to the brain and thereby to induce sickness behavior [47, 48], and therefore an influence of cytokines on pain perception is also possible.

In summary, our study suggests that cytokines during AMI are not only reacting to myocardial ischemic tissue damage, they are also connected to the subjective experience of the situation reflected by pain intensity. We found an association between acute pain levels and IL-33 and TGF- $\beta, 2$ predominantly anti-inflammatory cytokines, in a cohort of patients with AMI. To the best of our knowledge, the currently available evidence does not allow a firm statement as to the clinical relevance in terms of increased morbidity and mortality of the observed relationships between pain and cytokine levels. However, as IL-33 has well-established atheroprotective functions and is beneficial for cardiac remodeling, its decrease in conjunction with pain might worsen the prognosis of patients with cardiac disease.

\section{Disclosure Statement}

The authors declare that there is no conflict of interest.

\section{Funding Sources}

This research was supported by grant 140960 from the Swiss National Science Foundation to R.v.K. (PI), J.-P.S., U.S., H.Z., and J.B.

\section{References}

1 Whitehead DL, Strike P, Perkins-Porras L, Steptoe A: Frequency of distress and fear of dying during acute coronary syndromes and consequences for adaptation. Am J Cardiol 2005;96:1512-1516.

2 Ginzburg K, Solomon Z, Koifman B, Keren G, Roth A, Kriwisky M, et al: Trajectories of posttraumatic stress disorder following myocardial infarction: a prospective study. J Clin Psychiatry 2003;64:1217-1223.

3 Hari R, Begré S, Schmid J-P, Saner H, Gander $M-L$, von Känel R: Change over time in posttraumatic stress caused by myocardial infarction and predicting variables. J Psychosom Res 2010;69:143-150.

4 Whitehead DL, Perkins-Porras L, Strike PC, Steptoe A: Post-traumatic stress disorder in patients with cardiac disease: predicting vulnerability from emotional responses during admission for acute coronary syndromes. Heart 2006;92:1225-1229.

5 Wiedemar L, Schmid J-P, Müller J, Wittmann L, Schnyder U, Saner H, et al: Prevalence and predictors of posttraumatic stress disorder in patients with acute myocardial infarction. Heart Lung 2008;37:113-121.
6 von Känel R, Hari R, Schmid J-P, Saner H, Begré S: Distress related to myocardial infarction and cardiovascular outcome: a retrospective observational study. BMC Psychiatry 2011;11:98.

7 Frangogiannis NG: Regulation of the inflammatory response in cardiac repair. Circ Res 2012:110:159-173.

8 Prabhu SD, Frangogiannis NG: The biological basis for cardiac repair after myocardial infarction: from inflammation to fibrosis. Circ Res 2016;119:91-112.

9 Dobaczewski M, Chen W, Frangogiannis NG: Transforming growth factor (TGF) $-\beta$ signaling in cardiac remodeling. J Mol Cell Cardiol 2011;51:600-606

10 Seki K, Sanada S, Kudinova AY, Steinhauser ML, Handa V, Gannon J, et al: Interleukin33 prevents apoptosis and improves survival after experimental myocardial infarction through ST2 signaling. Circ Heart Fail 2009; 2:684-691.

11 Kunes P, Mandak J, Holubcova Z, Kolackova $\mathrm{M}$, Krejsek J: Actual position of interleukin (IL)-33 in atherosclerosis and heart failure: great expectations or en attendant Godot? Perfusion 2015;30:356-374.
12 Kuebler U, Zuccarella-Hackl C, Arpagaus A, Wolf JM, Farahmand F, von Känel R, et al: Stress-induced modulation of NF- $\mathrm{KB}$ activation, inflammation-associated gene expression, and cytokine levels in blood of healthy men. Brain Behav Immun 2015;46:87-95.

13 Marsland AL, Walsh C, Lockwood K, JohnHenderson NA: The effects of acute psychological stress on circulating and stimulated inflammatory markers: a systematic review and meta-analysis. Brain Behav Immun 2017; 64:208-219.

14 Steptoe A, Hamer M, Chida Y: The effects of acute psychological stress on circulating inflammatory factors in humans: a review and meta-analysis. Brain Behav Immun 2007;21: 901-912.

15 von Känel R, Mills PJ, Mausbach BT, Dimsdale JE, Patterson TL, Ziegler MG, et al: Effect of Alzheimer caregiving on circulating levels of C-reactive protein and other biomarkers relevant to cardiovascular disease risk: a longitudinal study. Gerontology 2012;58:354-365.

16 Pace TWW, Heim CM: A short review on the psychoneuroimmunology of posttraumatic stress disorder: from risk factors to medical comorbidities. Brain Behav Immun 2011;25:6-13. 
17 Passos IC, Vasconcelos-Moreno MP, Costa LG, Kunz M, Brietzke E, Quevedo J, et al: Inflammatory markers in post-traumatic stress disorder: a systematic review, meta-analysis, and meta-regression. Lancet Psychiatry 2015; 2:1002-1012.

18 von Känel R, Begré S, Abbas CC, Saner H, Gander M-L, Schmid J-P: Inflammatory biomarkers in patients with posttraumatic stress disorder caused by myocardial infarction and the role of depressive symptoms. Neuroimmunomodulation 2010;17:39-46.

19 von Känel R, Hepp U, Kraemer B, Traber R, Keel M, Mica L, et al: Evidence for low-grade systemic proinflammatory activity in patients with posttraumatic stress disorder. J Psychiatr Res 2007;41:744-752.

20 Reuben DB, Cheh AI, Harris TB, Ferrucci L, Rowe JW, Tracy RP, et al: Peripheral blood markers of inflammation predict mortality and functional decline in high-functioning community-dwelling older persons. J Am Geriatr Soc 2002;50:638-644.

21 Tuomisto K, Jousilahti P, Sundvall J, Pajunen $\mathrm{P}$, Salomaa V: C-reactive protein, interleukin- 6 and tumor necrosis factor alpha as predictors of incident coronary and cardiovascular events and total mortality. A populationbased, prospective study. Thromb Haemost 2006;95:511-518.

22 Wassel CL, Barrett-Connor E, Laughlin GA: Association of circulating C-reactive protein and interleukin- 6 with longevity into the 80 s and 90s: the Rancho Bernardo Study. J Clin Endocrinol Metab 2010;95:4748-4755.

23 Ridker PM, Rifai N, Pfeffer M, Sacks F, Lepage S, Braunwald E: Elevation of tumor necrosis factor-alpha and increased risk of recurrent coronary events after myocardial infarction. Circulation 2000;101:2149-2153.

24 Hartford M, Wiklund O, Mattsson Hultén L, Persson A, Karlsson T, Herlitz J, et al: C-reactive protein, interleukin-6, secretory phospholipase $A_{2}$ group IIA and intercellular adhesion molecule-1 in the prediction of late outcome events after acute coronary syndromes. J Intern Med 2007;262:526-536.

25 Kavsak PA, Ko DT, Newman AM, Palomaki GE, Lustig V, MacRae AR, et al: Risk stratification for heart failure and death in an acute coronary syndrome population using inflammatory cytokines and $\mathrm{N}$-terminal pro-brain natriuretic peptide. Clin Chem 2007;53: 2112-2118

26 Zamani P, Schwartz GG, Olsson AG, Rifai N, Bao W, Libby P, et al: Inflammatory biomarkers, death, and recurrent nonfatal coronary events after an acute coronary syndrome in the MIRACL study. J Am Heart Assoc 2013; 2:e003103.
27 Demyanets S, Speidl WS, Tentzeris I, Jarai R, Katsaros KM, Farhan S, et al: Soluble ST2 and interleukin-33 levels in coronary artery disease: relation to disease activity and adverse outcome. PLoS One 2014;9:e95055.

28 Dhillon OS, Narayan HK, Khan SQ, Kelly D, Quinn PA, Squire IB, et al: Pre-discharge risk stratification in unselected STEMI: is there a role for ST2 or its natural ligand IL-33 when compared with contemporary risk markers? Int J Cardiol 2013;167:2182-2188.

29 Tashiro H, Shimokawa H, Sadamatu K, Yamamoto K: Prognostic significance of plasma concentrations of transforming growth factor-beta in patients with coronary artery disease. Coron Artery Dis 2002;13:139-143.

30 Meister R, Princip M, Schmid J-P, Schnyder U, Barth J, Znoj H, et al: Myocardial Infarction - Stress PRevention INTervention (MISPRINT) to reduce the incidence of posttraumatic stress after acute myocardial infarction through trauma-focused psychological counseling: study protocol for a randomized controlled trial. Trials 2013;14:329.

31 Fox KAA, Dabbous OH, Goldberg RJ, Pieper KS, Eagle KA, Werf FV de, et al: Prediction of risk of death and myocardial infarction in the six months after presentation with acute coronary syndrome: prospective multinational observational study (GRACE). BMJ 2006;333: 1091.

32 Killip T, Kimball JT: Treatment of myocardial infarction in a coronary care unit. A two year experience with 250 patients. Am J Cardiol 1967;20:457-464.

33 Price DD, McGrath PA, Rafii A, Buckingham $B$ : The validation of visual analogue scales as ratio scale measures for chronic and experimental pain. Pain 1983;17:45-56.

34 Bryant RA, Moulds ML, Guthrie RM: Acute stress disorder scale: a self-report measure of acute stress disorder. Psychol Assess 2000;12: 61-68.

35 Helfricht S, Landolt MA, Moergeli H, Hepp U, Wegener D, Schnyder U: Psychometric evaluation and validation of the German version of the Acute Stress Disorder Scale across two distinct trauma populations. J Trauma Stress 2009;22:476-480.

36 American Psychiatric Association: Diagnostic and Statistical Manual of Mental Disorders, ed 4. Washington, American Psychiatric Association, 1994.

37 Do CB, Batzoglou S: What is the expectation maximization algorithm? Nat Biotechnol 2008;26:897-899.
38 Dhillon OS, Narayan HK, Quinn PA, Squire IB, Davies JE, Ng LL: Interleukin 33 and ST2 in non-ST-elevation myocardial infarction: comparison with Global Registry of Acute Coronary Events Risk Scoring and NT-proBNP. Am Heart J 2011;161:1163-1170.

39 Zhang K, Zhang X, Mi Y, Liu J: Predicting value of serum soluble ST2 and interleukin-33 for risk stratification and prognosis in patients with acute myocardial infarction. Chin Med J (Engl) 2013;126:3628-3631.

40 Rath D, Chatterjee M, Müller I, Müller K, Böckmann C, Droppa M, et al: Platelet expression of transforming growth factor beta 1 is enhanced and associated with cardiovascular prognosis in patients with acute coronary syndrome. Atherosclerosis 2014;237:754759.

41 Lantero A, Tramullas M, Díaz A, Hurlé MA: Transforming growth factor- $\beta$ in normal nociceptive processing and pathological pain models. Mol Neurobiol 2012;45:76-86.

42 Pervanidou P, Kolaitis G, Charitaki S, Margeli A, Ferentinos S, Bakoula C, et al: Elevated morning serum interleukin (IL)-6 or evening salivary cortisol concentrations predict posttraumatic stress disorder in children and adolescents six months after a motor vehicle accident. Psychoneuroendocrinology 2007;32: 991-999.

43 Marques-Vidal P, Bochud M, Bastardot F, Lüscher T, Ferrero F, Gaspoz J-M, et al: Levels and determinants of inflammatory biomarkers in a Swiss population-based sample (CoLaus study). PLoS One 2011;6:e21002.

44 Liu Y, Ho RC-M, Mak A: Interleukin (IL)-6, tumour necrosis factor alpha (TNF- $\alpha$ ) and soluble interleukin-2 receptors (sIL-2R) are elevated in patients with major depressive disorder: a meta-analysis and meta-regression. J Affect Disord 2012;139:230-239.

45 Tuglu C, Kara SH, Caliyurt O, Vardar E, Abay E: Increased serum tumor necrosis factor-alpha levels and treatment response in major depressive disorder. Psychopharmacology (Berl) 2003;170:429-433.

46 Sheps DS, Creed F, Clouse RE: Chest pain in patients with cardiac and noncardiac disease. Psychosom Med 2004;66:861-867.

47 Kronfol Z, Remick DG: Cytokines and the brain: implications for clinical psychiatry. Am J Psychiatry 2000;157:683-694.

48 Maier SF, Watkins LR: Cytokines for psychologists: implications of bidirectional immuneto-brain communication for understanding behavior, mood, and cognition. Psychol Rev 1998;105:83-107. 